\title{
Colonic Gene Expression and Fecal Microbiota in Diarrhea-predominant Irritable Bowel Syndrome: Increased Toll-like Receptor 4 but Minimal Inflammation and no Response to Mesalazine
}

\author{
Jonna Jalanka, ${ }^{1,2}$ Ching Lam, ${ }^{2}$ Andrew Bennett, ${ }^{2,3}$ Anna Hartikainen, ${ }^{1}$ Fiona Crispie, ${ }^{4,5}$ Laura A Finnegan, ${ }^{4,5}$ Paul D Cotter, \\ and Robin Spiller ${ }^{2 *}$
}

${ }^{1}$ Human Microbiome Research Program, Faculty of Medicine, University of Helsinki, Helsinki, Finland; ${ }^{2}$ Nottingham Digestive Diseases Center and NIHR Nottingham Biomedical Research Center at Nottingham University Hospitals NHS Trust, the University of Nottingham, Nottingham, Notts, UK; ${ }^{3}$ FRAME Alternatives Laboratory, School of Life Sciences, University of Nottingham, Medical School, QMC, Nottingham, Notts, UK; ${ }^{4}$ Teagasc Food Research Center, Moorepark, Fermoy, Co. Cork, Ireland; and ${ }^{5}$ APC Microbiome Ireland, Cork, Ireland

\section{Background/Aims}

Diarrhea-predominant irritable bowel syndrome (IBS-D) has been previously associated with evidence of immune activation and altered microbiota. Our aim is to assess the effect of the anti-inflammatory agent, mesalazine, on inflammatory gene expression and microbiota composition in IBS-D.

\section{Methods}

We studied a subset of patients $(n=43)$ from a previously published 12-week radomized placebo-controlled trial of mesalazine. Mucosal biopsies were assessed by immunohistochemistry and reverse transcription-polymerase chain reaction for a range of markers of inflammation, altered permeability, and sensory receptors including Toll-like receptors (TLRs) at randomization after treatment. All biopsy data were compared to 21 healthy controls. Patient's stool microbiota composition was analysed through 165 ribosomal RNA sequencing.

\section{Results}

We found no evidence of increased immune activation compared to healthy controls. However, we did find increased expression of receptors in both sensory pathways and innate immune response including TLR4. Higher TLR4 expression was associated with greater urgency. TLR4 expression correlated strongly with the expression of the receptors bradykinin receptor B2, chemerin chemokine-like receptor 1, and transient receptor potential cation channel, subfamily A, member 1 as well as TLR4's downstream adaptor myeloid differentiation factor 88. Mesalazine had minimal effect on either gene expression or microbiota composition.

\section{Conclusions}

Biopsies from a well-characterized IBS-D cohort showed no substantial inflammation. Mesalazine has little effect on gene expression and its previous reported effect on fecal microbiota associated with much greater inflammation found in inflammatory bowel diseases is likely secondary to reduced inflammation. Increased expression of TLR4 and correlated receptors in IBS may mediate a general increase in sensitivity to external stimuli, particularly those that signal via the TLR system.

(J Neurogastroenterol Motil 2021;27:279-291)

Key Words

Gene expression; Irritable bowel syndrome; Mesalazine; Microbiota; Toll-like receptor 4 


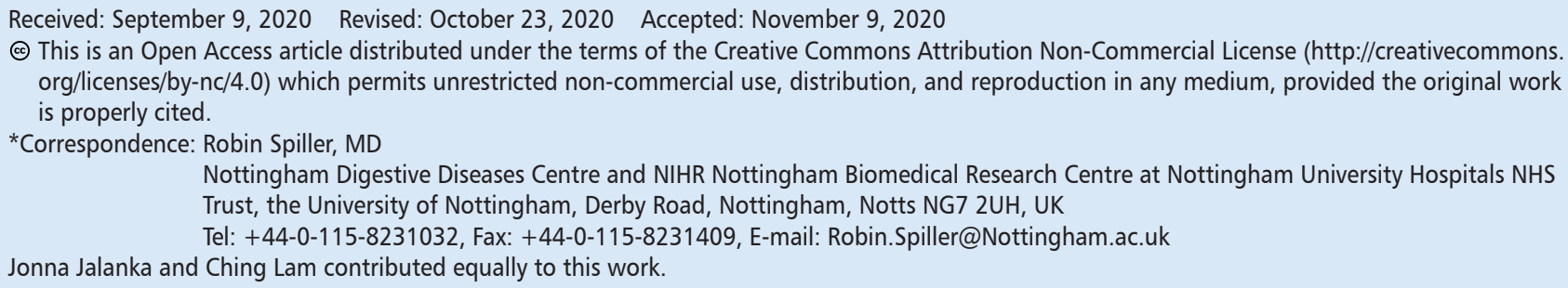

\section{Introduction}

Irritable bowel syndrome with diarrhea (IBS-D) is a common condition, affecting up to $1 \%$ of the population, characterized by frequent loose stools, abdominal cramps and urgency which considerably impair the quality of life. Clinical diagnosis requires the exclusion of a range of inflammatory conditions which closely mimic IBS-D, including microscopic colitis ${ }^{1}$ and Crohn's disease, as well as bile salt malabsorption which is recently recognized to account for approximately 1 in 4 of patients presenting with symptoms of IBS-D. ${ }^{2}$ IBS-D can also develop after a bout of gastroenteritis, socalled post-infectious IBS (PI-IBS). ${ }^{3}$ The risk of developing PIIBS is increased by the presence of markers of severity such as fever and rectal bleeding, suggesting that the resulting immune activation is a key factor in IBS symptoms. ${ }^{4}$ Both PI-IBS and other inflammatory conditions are associated with distinctive changes in microbiota. ${ }^{5-7}$ The commonest causes of PI-IBS are infections with either Campylobacter, Salmonella, Shigella, and Vibrio cholerae, all members of the Proteobacteria, which produce many toxins including lipopolysaccharide (LPS) that act through Toll-like receptor 4 (TLR4) to activate the cytokine cascade typical of infectious gastroenteritis.

Pilot data had suggested that mesalazine, through its antiinflammatory actions, could normalize the underlying immune activation and impaired barrier function described in both PIIBS $^{3,8}$ and IBS-D. ${ }^{9-11}$ The current work describes the baseline host gene expression of a range of markers of inflammation, altered permeability, and sensory receptors including TLRs along with the microbiota composition in IBS-D and their response to the antiinflammatory agent mesalazine. Mesalazine has been reported to reduce "dysbiosis" of the microbiota in patients with inflammatory bowel diseases (IBD), reducing the abundance of Proteobacteria such as Escherichia-Shigella while increasing Enterococcus, Lactococcus, and Lactobacillus. ${ }^{12,13}$ It is uncertain if these changes are secondary to the reduction in inflammation and diarrhea or a direct effect of mesalazine. Mesalazine can inhibit polyphosphate kinase (PPK) activity, which would make the intestinal microbiome more sensitive to oxidative stress, reduce colonization, and diminish the dysbiotic microbiotas' ability to persist in the inflamed gut. ${ }^{14}$ The bacteria targeted by this mechanism include Proteobacteria and Bacteroidetes, species often reported to be increased in the inflamed intestine of patients with IBD. ${ }^{12,15}$ However, inflammation on its own is known to alter the microbiota composition, eg, by increasing the colonic oxygen levels which allows facultative anaerobes, often reported to be increased in patients with IBD, to flourish. ${ }^{16,17}$ The exact effect of mesalazine on the intestinal microbiota is uncertain, with one small interventional study in IBS showing a reduction of the bacterial load by almost $50 \%{ }^{18}$ while larger cross-sectional population studies showed very little change as a consequence of taking mesalazine. ${ }^{19,20}$

Our aim is, therefore, to take advantage of a group of wellcharacterized patients with IBS-D about to enter a clinical trial of mesalazine to determine (1) if inflammatory gene expression was increased, (2) whether this was ameliorated by mesalazine treatment, and (3) if mesalazine intervention would alter fecal microbiota and their associations with gene expression in IBS-D.

\section{Materials and Methods}

\section{Study Design}

This multi-centered, parallel group, randomized placebocontrolled trial of mesalazine $2 \mathrm{~g}$ twice daily for treatment of IBS$\mathrm{D}$ has been previously reported..$^{21}$ Full details are available in the publication, but in brief, 115 patients meeting the Rome III criteria for IBS-D were randomized to receive either mesalazine or placebo for 12 weeks. Known causes of diarrhea were excluded by requiring patients to have a normal colonoscopy and a biopsy excluding microscopic colitis and to have either a normal selenium homocholic acid taurine (SeHCAT) test or negative response to cholestyramine. We also excluded lactose intolerance by means of a lactose challenge of $568 \mathrm{~mL}$ of skimmed milk which the patient performed at home. If any symptoms developed, then we performed a breath hydrogen lactose tolerance test using $25 \mathrm{~g}$ of lactose in the hospital to confirm the finding. A small number of patients met criteria ${ }^{7}$ for PI-IBS. 
Table 1. Demographics of Patients With Irritable Bowel Syndrome and Healthy Controls

\begin{tabular}{lccc}
\hline Demographics & $\begin{array}{c}\mathrm{HC} \\
(\mathrm{n}=21)\end{array}$ & $\begin{array}{c}\text { Mesalazine } \\
(\mathrm{n}=21)\end{array}$ & $\begin{array}{c}\text { Placebo } \\
(\mathrm{n}=22)\end{array}$ \\
\hline Age $(\mathrm{yr})$ & $42.8(20-75)$ & $38.0(19-65)$ & $43.7(22-69)$ \\
Sex (F/M) & $16 / 5$ & $13 / 8$ & $13 / 9$ \\
Anxiety & $5.20(2.74)$ & $7.86(4.50)$ & $8.00(3.59)$ \\
Depression & $1.60(1.29)$ & $4.67(4.04)$ & $4.05(2.98)$ \\
Total HADS & $6.80(3.41)$ & $12.52(7.51)$ & $12.05(5.81)$ \\
PHQ-12 SS & $2.15(1.84)$ & $6.67(5.17)$ & $4.15(3.03)$ \\
\hline
\end{tabular}

HC, healthy controls; F, female; M, male; HADS, Hospital Anxiety and Depression Scale; PHQ-12 SS, Patient Health Questionnaire-12 Somatic Symptom score.

Anxiety and depression assessed using the HADS and PHQ-12 SS score. Values are expressed as mean (range), n, or mean (SD).

After documenting psychological symptoms using the Hospital Anxiety and Depression Scale (HADS) along with the Patient Health Questionnaire-12 Somatic Symptom (PHQ-12 SS) score, bowel frequency, stool consistency, and bowel symptoms (pain and urgency) were recorded daily in a diary over a 14-day period. Pain severity was recorded on a 0-10 scale (none- the most severe pain ever experienced) along with pain duration in hours/day, bloating on a 0-10 scale and the presence of urgency each day (yes/no). Symptoms were recorded each evening to summarize the preceding 24 hours.

The subgroup of patients ( $n=43$; Table 1$)$, whose data is reported here, were those recruited in the Nottingham center who consented for sigmoid biopsy and stool samples prior to randomization and at the end of the 12-week trial of either mesalazine or placebo. We also obtained sigmoid biopsies in 21 healthy volunteers, recruited as controls for a previous trial. ${ }^{22}$ Biopsies were obtained at $30 \mathrm{~cm}$ from the anus during an unprepared, unsedated, flexible sigmoidoscopy in the left lateral position. Biopsies were taken and processed for either immunohistochemistry or incubated in RNAlater at $4^{\circ} \mathrm{C}$ for 24 hours before storing frozen at $-80^{\circ} \mathrm{C}$ prior to assay. Stool samples from the patients were collected less than 2 hours prior to storing at $-80^{\circ} \mathrm{C}$. Stool samples were not collected from the healthy controls in the previous trial. The study was approved by the National Research Ethics Service (Research Ethics Committee reference No. 10/H0605/65) and by the Medicines and Healthcare Regulatory Authority (CTA reference 03057/0046/001-0001). The trial was registered on ClinicalTrial.gov as ISRCTN76612274. No change was made to the endpoints after registration.

\section{Laboratory Methods}

\section{Immunohistochemistry}

Formalin-fixed and paraffin-embedded $4 \mu \mathrm{m}$ sections were mounted on precoated slides and following dewaxing, underwent routine $\mathrm{H} \& \mathrm{E}$ staining as well as immunostaining with antibodies, all from Dako, Glostrup, Denmark. These included CD68 (macrophage marker) and mast cell tryptase (mast cell marker). Slides were processed using the Dako Techmate 500 Plus and Techmate reagents as previously described. ${ }^{21}$

\section{Image analysis}

Slides were prepared and scanned into the computer using a nanozoomer displayed at $\times 40$ magnification. Mast cell tryptase expression was detected in the lamina propria using automatic software (i-Tem Desktop by Olympus, Hamburg, Germany) as some mast cells were in a degranulated state, making individual cell counting difficult. Detection of MCT stained cell was checked for reproducibility ( $>90 \%$ ) using a standard specimen before cell counting began. Results were presented as the percentage area stained for mast cell tryptase. CD68 cells were counted in 5 high power fields and expressed per $\mathrm{mm}^{2}$.

\section{Host gene expression}

RNA purification. RNA was extracted from sigmoid colorectal tissue biopsy and stored in RNAlater, at $-80^{\circ} \mathrm{C}$. RNA extraction was performed using TRI reagent (Pcode101078497 T9424; Sigma Aldrich, St. Louis, MO, USA) followed by cleanup of RNA using a Qiagen RNeasy column (Cat No 74106; Qiagen, Valencia, CA, USA). Synthesis of cDNA was performed using Superscript 3 reverse transcriptase according to manufacturer's protocols.

Gene card. Custom-made 96 gene micro-fluidic gene cards (Format 96a, P/N 4342259; Applied Biosystems, Foster City, CA, USA) were designed to include genes associated with chronic inflammation, altered barrier function and nociception in IBS and IBD. Reference genes, which were chosen to be consistently expressed during the trial duration, included 18S RNA, beta-actin, ribosomal protein large $\mathrm{PO}$, and hypoxanthine phosphoribosyltransferase 1 . Gene expression values were calculated as arbitrary units using the geometric mean of the reference genes described above and calculating the $2^{\Delta \mathrm{Ct}}$ for each gene. ${ }^{23} \mathrm{~A}$ full list of genes, their abbreviations and full technical details can be found in the Supplementary Table 1 and Supplementary Method. 


\section{Microbiota analysis}

The stool microbiota DNA was extracted with a validated method using both mechanical as well as chemical lysis to breakdown bacterial cells. ${ }^{24,25}$ The V3-V4 hypervariable region of the $16 \mathrm{~S}$ rRNA gene was amplified and sequenced with the Illumina MiSeq platform using the standard Illumina protocol. ${ }^{26}$ As a positive sequencing control, we used the ZymoBIOMICS Microbial Community Standard (Cat. No. D6306; Zymo Research, Irvine, CA, USA) and all DNA extraction controls were sequenced to control for potential reagent contaminations. The composition of the standard sequence replicated what was reported by the provider and the extraction negative controls contained less than 4000 reads per sample, containing known reagent contaminants. The obtained sequence reads (on average 131166 per sample) were prepossessed with Mare R package. ${ }^{27}$ In brief, the forward reads were trimmed to $180 \mathrm{nt}$ length and to exclude sequencing errors, rare reads were excluded by quality filtering reads with low abundance. Chimeras were filtered and reads were clustered to amplicon sequence variant before taxonomical assignment using USEARCH (version 8.1.1756) and SILVA 16S rRNA reference database version 115. After pre-processing, there were on average 66718 reads per sample (ranging from 21739 to 125417 ).

\section{Statistical Methods}

All statistical methods were conducted with $\mathrm{R}$ (version 3.6.1). The histology and gene expression data were tested for normal distribution with the Shapiro-Wilk test. The significance between patients with IBS-D and healthy controls histology and gene expression was assessed by the Mann-Whitney $U$ test and corrected for multiple testing with Bonferroni correction. Correlations and the significance of the association between gene expression and symptoms were measured with Spearman correlations and the statistical significance of the correlations was corrected for multiple testing with the false discovery rate (FDR) approach. Correlations over 0.35 and with $q$-values less than 0.01 were considered to be significant. The division of samples into those with high and low TLR4 expression was set to be mean plus 1.5 times the standard deviation of the expression of the healthy controls as explained in Supplementary Figure 1.

For microbiota composition analysis, we used the following $\mathrm{R}$ packages for the microbiota analysis; mare, ${ }^{27}$ vegan, ${ }^{28} \mathrm{MASS}^{29}$ and nlme. $^{28}$ All taxonomic ranks down to genus level taxa were used for statistical testing. Microbial alpha diversity was assessed using inverse Simpson diversity index using operational taxonomic unit level data. Principal coordinate analysis with Bray-Curtis dissimilarities was used to visualize microbial beta diversity. To test differences in the bacterial abundance between study groups and the associations between the bacterial taxa and gene expression, generalized linear mixed models were used. In the models the read number for each sample was used as an offset and subjects' age and sequencing batch were used as confounding factors. The obtained $P$-values were adjusted for multiple testing with the FDR approach, and FDR-adjusted $P$-values ( $q$-values) below 0.050 were considered to be significant.

\section{Results}

\section{Baseline Comparison Between Healthy Controls and Patients With Diarrhea-predominant Irritable Bowel Syndrome}

Our patients were typical of other IBS patient series, being predominantly middle-aged females with mildly elevated anxiety and depression scores and increased non-gastroenterological symptoms as assessed by the PHQ-12 SS (Table 1). The PHQ was > 6 (upper limit of normal $^{30}$ ) in $46.9 \%$ of the patients but none of the controls. While $51.1 \%$ scored $>7$ (upper limit of normal) on the anxiety scale only $32.7 \%$ were abnormal on the depression scale. As required for trial entry, the patients had frequent loose stools associated with bloating and abdominal pain (Table 2). Healthy controls were well matched with the patients for age and gender. All were pain-free with stool frequency median (interquartile range) of 1.0 (1.0-1.4) /day and none met the Rome III criteria. As previously reported $^{20}$ there were no significant differences between mesalazine and placebo subjects with regard to the primary endpoint, daily average stool frequency, which was $(2.8 ; \mathrm{SD}, 1.2)$ on mesalazine and (2.7; SD, 1.9) on placebo with adjusted between-group difference of 0.1 ( $95 \% \mathrm{CI},-0.33$ to $0.53 ; P=0.660)$. Neither were there

Table 2. Gastrointestinal Symptoms of Patients at Randomization

\begin{tabular}{lcc}
\hline \multicolumn{1}{c}{ GI symptoms } & Mesalazine & Placebo \\
\hline Abdominal pain severity score & $4.64(1.53)$ & $3.32(2.03)$ \\
Urgency & $6.04(1.65)$ & $5.23(2.03)$ \\
Bloating & $3.71(2.28)$ & $3.42(2.54)$ \\
Stool frequency/day & $4.10(1.72)$ & $3.87(2.21)$ \\
Stool consistency & $5.44(0.68)$ & $5.36(0.87)$ \\
\hline
\end{tabular}

GI, gastrointestinal.

Abdominal pain and bloating were assessed on 1-10 scale, urgency is days per week with urgency. Stool consistency assessed using Bristol Stool Form Score. Values are expressed as mean (SD). 
Table 3. Significant Gene Expression Differences Between Healthy Controls and Subjects With Diarrhea-predominant Irritable Bowel Syndrome at Baseline

\begin{tabular}{|c|c|c|c|c|}
\hline Gene & $\mathrm{HC}$ & IBS-D & $\begin{array}{l}\text { Fold change } \\
\text { (HC/IBS-D) }\end{array}$ & $P$-value \\
\hline ATP12A & $3.52(5.54)$ & $0.32(0.51)$ & 10.93 & 0.001 \\
\hline BDKRB2 & $10.37(3.35)$ & $17.06(6.39)$ & 0.61 & $<0.001$ \\
\hline CMKLR1 & $0.77(0.27)$ & $1.31(0.62)$ & 0.59 & 0.003 \\
\hline F2RL2 & $0.08(0.027)$ & $0.17(0.09)$ & 0.50 & 0.001 \\
\hline F2RL3 & $0.29(0.14)$ & $0.51(0.25)$ & 0.57 & 0.006 \\
\hline FABP2 & $2.93(1.25)$ & $6.19(2.75)$ & 0.47 & $<0.001$ \\
\hline FST & $0.06(0.06)$ & $0.11(0.08)$ & 0.52 & 0.010 \\
\hline IL-37 & $0.53(0.25)$ & $0.33(0.23)$ & 1.58 & 0.016 \\
\hline MMP2 & $8.31(2.99)$ & $12.90(5.14)$ & 0.64 & 0.007 \\
\hline PTGS1 & $2.95(1.00)$ & $4.20(1.61)$ & 0.70 & 0.036 \\
\hline TBXAS1 & $19.46(51.61)$ & $4.45(2.20)$ & 4.37 & 0.042 \\
\hline TGFBR1 & $21.31(79.05)$ & $4.69(1.53)$ & 4.55 & 0.049 \\
\hline TJP1 & $7.13(2.95)$ & $10.35(3.08)$ & 0.69 & 0.009 \\
\hline TLR4 & $1.49(0.55)$ & $2.56(0.80)$ & 0.58 & $<0.001$ \\
\hline TPH1 & $24.75(44.51)$ & $7.48(3.01)$ & 3.31 & 0.020 \\
\hline TRPA1 & $7.13(11.02)$ & $8.12(3.84)$ & 0.88 & $<0.001$ \\
\hline
\end{tabular}

HC, healthy controls; IBS-D, diarrhea-predominant irritable bowel syndrome. Gene expression measurements were calculated as $2^{\Delta \mathrm{Ct}}$ using the geometric mean of 4 reference genes described in methods and values are given as arbitrary units.

For gene abreviations please see Supplementary Table 1.

Values are expressed as mean (SD).

$P$-values were corrected for multiple comparisons.

any differences in other clinical features including abdominal pain severity, average stool consistency and number of days with stool consistency type 6-7. ${ }^{21}$

\section{Histology}

All patient biopsies had been reviewed by a pathologist (Abed Zaitoun) and reported as within normal limits to exclude microscopic colitis prior to trial entry. Quantitative histology showed no evidence of inflammation. Mast cell percentage area stained was not elevated in these patients, mean (SD) being $2.43 \%(0.88)$ as compared to $2.26 \%(0.79)$ in the controls. There were no significant differences after 12 weeks of mesalazine $2.39 \%$ (0.69) as compared to placebo $2.20 \%$ (0.60). CD68 counts at baseline were slightly lower in the IBS-D at $1078(415) / \mathrm{mm}^{2}$ compared to healthy control values of $1404(364) / \mathrm{mm}^{2}, P=0.001$, but at 12 weeks, there were no significant differences between mesalazine and controls being 1062 (237) $/ \mathrm{mm}^{2}$ and $1029(183) / \mathrm{mm}^{2}$, respectively.
Table 4. Expression of Toll-like Receptors in Patients With Diarrheapredominant Irritable Bowel Syndrome at Baseline Compared to Healthy Controls

\begin{tabular}{cccr}
\hline Gene & HC & IBS-D & $P$-value \\
\hline TLR2 & $0.19(0.08)$ & $0.27(0.40)$ & 0.242 \\
TLR4 & $1.49(0.54)$ & $2.56(0.80)$ & $<0.001$ \\
TLR5 & $0.22(0.13)$ & $0.29(0.46)$ & 0.361 \\
TLR7 & $1.04(3.06)$ & $0.45(0.26)$ & 0.394 \\
TLR9 & $2.07(1.76)$ & $1.30(1.47)$ & 0.092 \\
\hline
\end{tabular}

HC, healthy controls; IBS-D, diarrhea-predominant irritable bowel syndrome; TLR, Toll-like receptor.

Values are expressed as mean (SD).

\section{Difference in the Host Gene Expression Between Healthy Controls and Patients With Diarrhea- predominant Irritable Bowel Syndrome}

We assessed a set of genes concerned with immune activation, altered barrier function and response to external stimuli which we believed would be altered in IBS-D and whose expression may be modulated by mesalazine. Contrary to our original hypothesis but in keeping with the lack of evidence of inflammation using conventional histology, we found little evidence that patients with IBS$\mathrm{D}$ had increased expression of key pro-inflammatory genes such as TNF- $\alpha$, IL-1, and IL-6 (Supplementary Table 1). Most of our target genes (76/92) showed no significant difference between healthy controls and patients with IBS-D at baseline, however, as shown in Table 3, several receptors including the protease activated receptors coagulation factor II [thrombin] receptor-like 2 (F2RL2), coagulation factor II [thrombin] receptor-like 3 (F2RL3), chemerin chemokine-like receptor 1 (CMKLR1), and bradykinin receptor B2 (BDKRB2), transient receptor potential cation channel, subfamily A, member 1 (TRPA1), tight junction protein 1 (TJP1), and TLR4 were significantly increased in IBS-D compared to our healthy controls.

\section{Expression of Toll-like Receptors in Diarrhea- predominant Irritable Bowel Syndrome}

Given the previous reports of increased TLR expression in IBS and patients with IBD, we assessed TLR 2, 4, 5, 7, and 9 expression in our patients at baseline which we compared with healthy controls; finding an increase in TLR4 only (Table 4 and Fig. 1). The mean gene expression value for TLR4 fell over the 12-week trial period in both groups, a greater numerical decrease was seen with mesalazine $(P=0.029)$ but the difference between treatment and placebo was not significant $(P=0.214$; Fig. $2 A)$. We found 
no effect on any of the other TLRs examined and downstream genes involved in TLR signalling such as MyD88 and nucleotidebinding oligomerization domain-containing protein 2 (NOD2) did not change significantly (Supplementary Fig. 2).

\section{Effect of Mesalazine on Gene Expression}

When comparing the gene expression of mesalazine treated patients with placebo after 12 weeks, we found no significant change in cytokines, prostaglandins, or cyclooxygenases, which is in keeping with the normal histology and lack of increased expression of established markers of inflammation (Supplementary Table 1). The only significant change in gene expression at the end of the trial be-

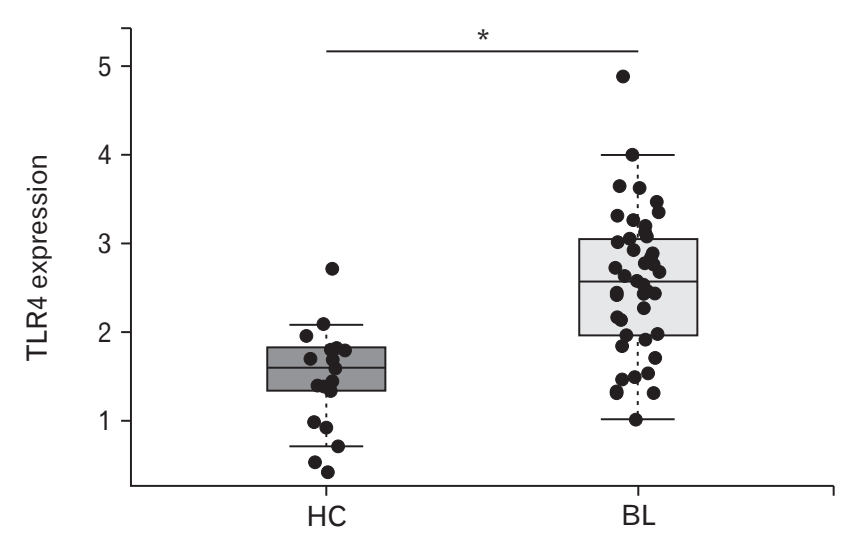

Figure 1. Toll-like receptor 4 expression in baseline (BL; $\mathrm{n}=44$ ) biopsy of patients with irritable bowel syndrome (IBS) is increased as compared to healthy controls ( $\mathrm{HC} ; \mathrm{n}=21)$. The line in the box shows the median expression value, boxes indicate the upper ( 75 th percentile) and lower quartiles (25th percentile) of the data, significant difference between $\mathrm{HC}$ and patients with diarrhea-predominant IBS is indicated with asterisk $\left({ }^{*} P<0.001\right)$.

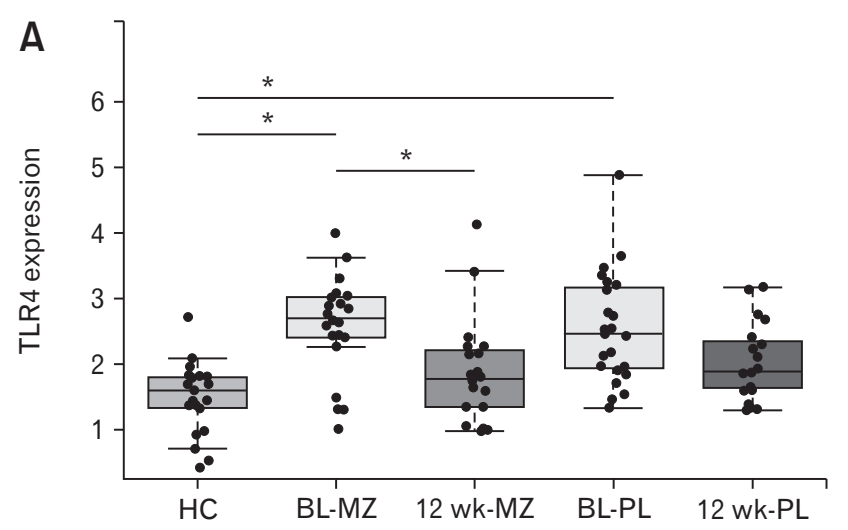

tween treatment and placebo was observed in TRPA1 $(P=0.018)$, which was depressed in the mesalazine treated group (Fig. 2B).

\section{Effect of Mesalazine Treatment on Fecal Microbiota Composition}

The mesalazine treatment had a minimal but statistically significant effect on the microbial composition accounting for just 4\% of the total microbial variation. Principal coordinate analysis based on the distance matrix of Bray-Curtis dissimilarity showed a statistical difference between the samples at baseline and after 12 weeks on mesalazine treatment ( $P=0.011$; Fig. 3$)$ whereas the placebo group was not significantly different from baseline. This observed difference was due to the decreased relative abundance of 9 genera (Table 5) within the mesalazine-treated group. Seven of these genera were present at a very low abundance and hence were unlikely to play a major role in overall microbiota function. The 2 more abundant genera that were significantly reduced after 12 weeks in the mesalazine treatment group were Flavonifractor and Sutterella. We also recorded an increase in the microbial richness in the placebo group after 12 weeks but not in the treated arm. We did not observe any statistical difference in microbial alpha diversity between the study groups.

\section{Associations Between Toll-like Receptor 4 Expression and Mucosal Gene-expression and Intestinal Microbiota}

We correlated TLR4 expression with the expression of other mucosal genes and computed linear models to find associations between intestinal microbiota abundances. We found that the TLR4 expression was significantly correlated with the expression of 17

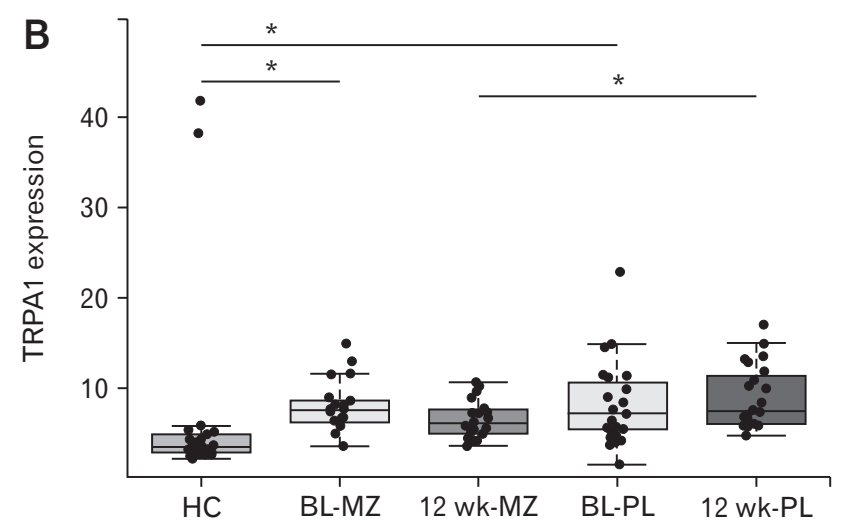

Figure 2. Effect of mesalazine (MZ) treatment on gene expression. Only 2 genes were affected by the mesalazine treatment. (A) The expression of Toll-like receptor 4 was significantly reduced in the treatment group after 12 weeks $(\mathrm{n}=21)$ as compared to baseline $(\mathrm{BL}$; $\mathrm{n}=22)$. (B) The transient receptor potential cation channel, subfamily A, member 1 (TRPA 1$)$ expression was significantly reduced after 12 weeks $(\mathrm{n}=21)$ when compared to the placebo $(\mathrm{PL})$ group $(\mathrm{n}=20)$. The significantly different expression is indicated with asterisk $\left({ }^{\star} P<0.05\right)$. HC, healthy controls. 

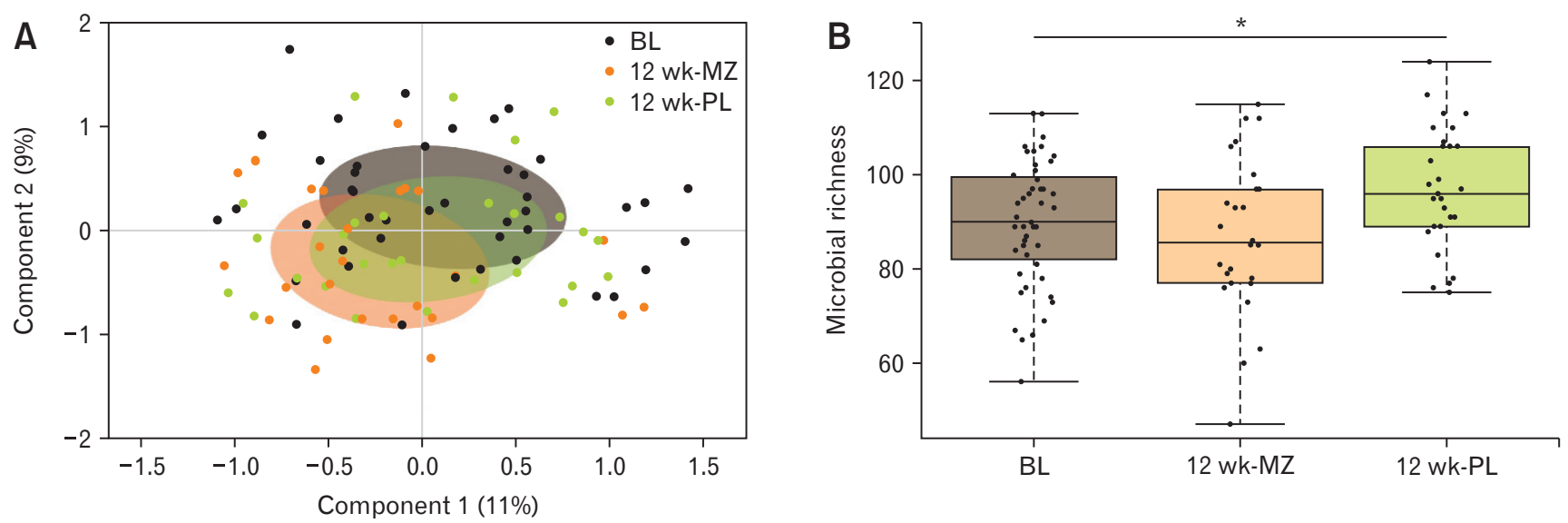

Figure 3. Effect of the trial on total microbiota composition $(n=98)$ measured with $(A)$ principal coordinate analysis based on the distance matrix of Bray-Curtis dissimilarity shows a statistical difference between the samples at baseline (BL) and after 12 weeks on mesalazine (MZ) treatment whereas the placebo (PL) group was not significantly different from BL, the colored circles represent $50 \%$ of the data. (B) There was a significant increase in microbial richness in the PL group but not MZ group after 12 weeks ( ${ }^{\star} P=0.011$ ).

Table 5. Genus Level Significant Differences Introduced by the Mesalazine Treatment

\begin{tabular}{|c|c|c|c|c|}
\hline \multirow{2}{*}{ Bacterial taxa } & \multicolumn{2}{|c|}{ Mesalazine } & \multicolumn{2}{|c|}{ Placebo } \\
\hline & BL & $12 \mathrm{wk}$ & BL & $12 \mathrm{wk}$ \\
\hline Firmicutes; Clostridia; Clostridiales; Family XIII Incertae Sedis; Incertae Sedis & 0.03 & $0.01^{\mathrm{a}}$ & 0.02 & 0.02 \\
\hline Firmicutes; Clostridia; Clostridiales; Heliobacteriaceae; Heliorestis & 0.04 & $0.02^{\mathrm{a}}$ & 0.01 & 0.01 \\
\hline Firmicutes; Clostridia; Clostridiales; Lachnospiraceae; Howardella & 0.04 & $0.01^{\mathrm{a}}$ & 0.05 & 0.04 \\
\hline Firmicutes; Clostridia; Clostridiales; Ruminococcaceae; Flavonifractor & 0.71 & $0.42^{\mathrm{a}}$ & 1.16 & 1.00 \\
\hline Firmicutes; Clostridia; Clostridiales; Ruminococcaceae; Saccharofermentans & 0.32 & $0.04^{\mathrm{a}}$ & $0.03^{\mathrm{a}}$ & $0.06^{\mathrm{a}}$ \\
\hline Proteobacteria; Alphaproteobacteria; Rhodospirillales; Rhodospirillaceae; Insolitispirillum & 0.06 & $0.01^{\mathrm{a}}$ & 0.06 & 0.04 \\
\hline Proteobacteria; Betaproteobacteria; Burkholderiales; Alcaligenaceae; Sutterella & 0.65 & $0.21^{\mathrm{a}}$ & 0.79 & 0.27 \\
\hline Proteobacteria; Deltaproteobacteria; Desulfuromonadales; Geobacteraceae; Geoalkalibacter & 0.10 & $0.01^{\mathrm{a}}$ & $0.01^{\mathrm{a}}$ & $0.01^{\mathrm{a}}$ \\
\hline Tenericutes; Mollicutes; Entomoplasmatales; Spiroplasmataceae; Spiroplasma & 0.03 & $0.00^{\mathrm{a}}$ & 0.04 & 0.02 \\
\hline
\end{tabular}

${ }^{a}$ Values indicate statistically significant difference as compared to treatment arm baseline (BL) sample.

Values show the relative abundance (\%) of each genus level taxon.

measured genes (Supplementary Table 2). The strongest correlations using both healthy controls and patients were those coding for the BDKRB2 $(r=0.58), \operatorname{MyD} 88(r=0.53)$, and CMKLR1 ( $r=0.47$ ) receptors, all $P<0.001$ (Fig. 4). TRPA1 was also significantly correlated with TLR4 expression $(r=0.46, P<0.001$; Fig. 4) and was uniquely significantly reduced by mesalazine treatment.

TLR4 expression was negatively associated with the microbial relative abundance of the Lactobacillus $(q=0.030)$ and Escherichia/Shigella genera $(q<0.001)$. There was a positive association detected with the relative abundance of the genera, Megasphera $(q=0.016)$ and Sutterella $(q=0.022)$, and the class Betaproteobacteria $(q=0.047)$.

\section{Link Between Gastrointestinal Symptoms and Toll- like Receptor 4 Expression}

In order to understand the relationship between TLR4 expression and patient symptoms, we subdivided the patients according to their TLR4 expression. The cut-off value was based on the TLR4 expression of the healthy controls (detailed in the Materials and Methods section). We showed that those patients with TLR4 above the cut-off at baseline experienced greater number of days with significant urgency during the 14 days recorded in the symptom diary when compared with those below the cut-off (Fig. 5). However, mesalazine treatment did not reduce the number of days with urgency, which actually rose slightly as previously reported in the 

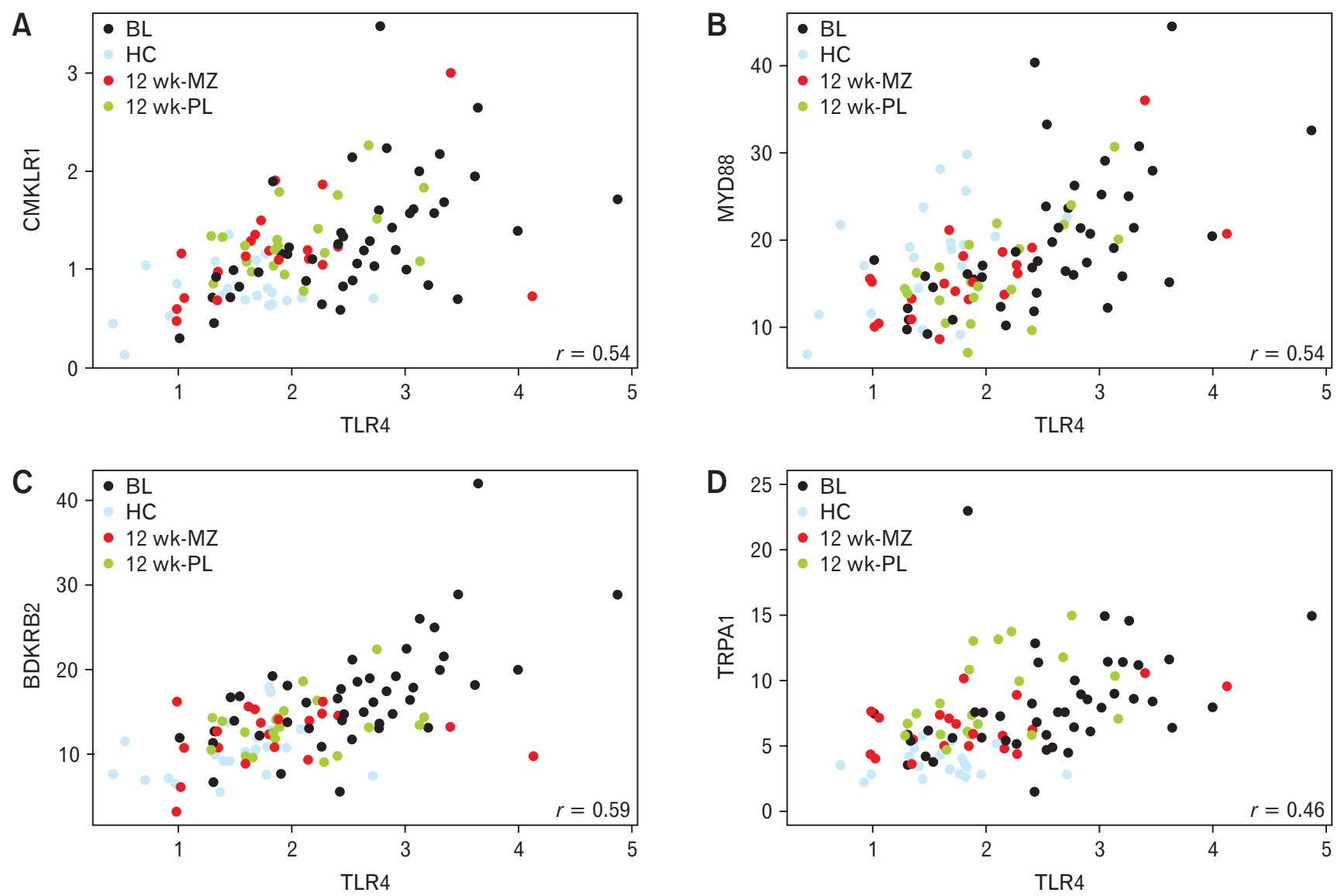

Figure 4. Correlations between Toll-like receptor 4 expression and gene expression of $(\mathrm{A})$ chemerin chemokine-like receptor 1 (CMKLR1; $r=$ 0.47), (B) myeloid differentiation factor 88 (Myd88; $r=0.53$ ), (C) bradykinin receptor B2 (BDKRB2; $r=0.58)$, and (D) transient receptor potential cation channel, subfamily A, member 1 (TRPA1; $r=0.46$ ), all $P<0.001$. BL, baseline; HC, healthy controls; MZ, mesalazine; PL, placebo.

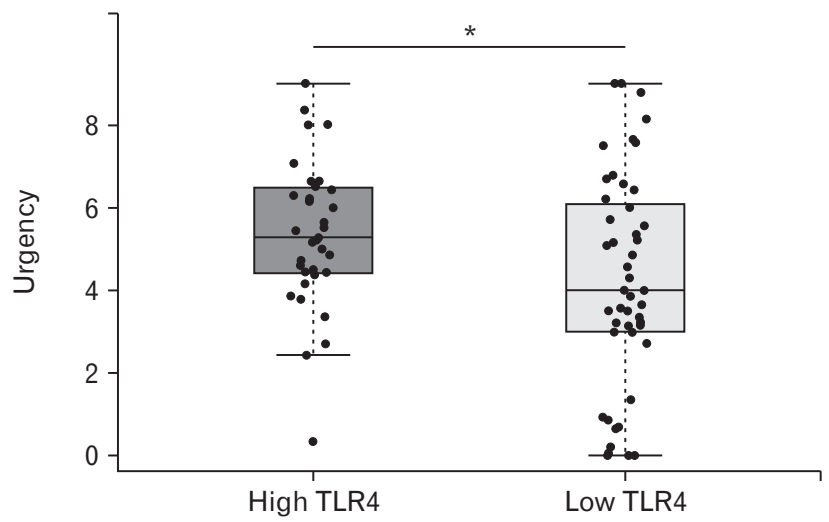

Figure 5. Days/week with urgency in patients divided according to Toll-like receptor 4 (TLR4) expression, high $=$ above threshold $(\mathrm{n}=$ $25)$, low $=$ below threshold $(\mathrm{n}=34)$. Urgency was significantly less frequent in subjects with low TLR4 expression than in those with high TLR4 expression (see Methods for the threshold estimation). Statistical significance indicated with asterisk $\left({ }^{*} P=0.054\right)$. main patient group. We found no evidence of a correlation between TLR4 and other symptoms including pain, stool frequency, or bloating $(P>0.05)$.

\section{Discussion}

Here we have shown that, contrary to our initial hypothesis, there was no evidence of substantial immune activation in the patients with IBS. While previous reports, including our own, ${ }^{3,31}$ have reported increases in both mast cells and cluster of differentiation 3 (CD3) lymphocytes, a meta-analysis ${ }^{32}$ suggests considerable heterogeneity, possibly because many of the studies have used unselected patients with IBS. Furthermore, few of the reports specifically exclude bile acid malabsorption and microscopic colitis as we did, which may possibly explain the differences from the current study. The other possible reason relates to the important role of psycho- 
logical distress, particularly depression, which has been shown to be associated with increased pro-inflammatory cytokines including TNF- $\alpha$ and IL-6. Our patients were chosen for their suitability for entering a clinical trial and few had significant depression, a fact which may also have reduced the pro-inflammatory tendency. None of the key pro-inflammatory genes such as TNF- $\alpha$, IL-1 $\beta$, and IL-6 were significantly increased in IBS in the current study compared to controls. An important limitation of our study and many previous studies was that the number of patients was relatively small, given the high degree of variability in gene expression. Using our data for IL-6 as an exemplar, at a power of $80 \%$ we could only detect a 2 -fold change in IL-6, the main reason being the large standard deviation of such measures, though it is worth noting that fold changes in IBD are much higher than this. We could not exclude smaller changes which may still have been significant. However, we confirmed other reports that the IBS mucosa does not show the type of cytokine activation seen in IBD where TNF- $\alpha$ expression for example is increased approximately 10 -fold. ${ }^{33}$ Our study differs somewhat from another recent study which found increased mucosa IL-6 (9-fold), along with TLR2, TLR4, and $\mathrm{C}-\mathrm{X}-\mathrm{C}$ motif chemokine ligand 11 (CXCL-11) and its receptor CXCR-3 ${ }^{34}$ However, we did find increased expression of a range of receptors including BDKRB 2, fatty acid binding protein 2 (FABP2), F2RL2, F2RL3, and CMKLR1 which rose 2-3 fold. This increase in receptors may be linked to a general increased sensitivity to proteases that have previously been shown to be increased in IBS-D stool ${ }^{35,36}$ and to mediate hypersensitivity to colorectal distension induced in mice by patient fecal supernatants. ${ }^{35}$ They also mediate neural activation by IBS mucosa supernatants. ${ }^{37}$ In addition, in contrast to previous reports, we detected only a very small effect of mesalazine on the intestinal microbiota composition, details of which are discussed below.

One of the limitations of our study was the relatively small number of subjects which meant that we could only detect relatively large changes as discussed above. The main trial reported earlier ${ }^{20}$ did not include healthy controls so we used additional heathy subjects who were recruited for another study, which did not require them to provide stool samples. We therefore could not benchmark the microbiota findings against healthy controls.

Our main trial, as previously reported, showed overall no benefit from mesalazine for either pain or bowel habit, ${ }^{21}$ a finding supported by a similar trial published the same year. ${ }^{38}$ Previous studies attempting to define either a general gene profile in IBS $^{39}$ or to test more specific hypotheses ${ }^{40-42}$ have failed to produce consistent findings, no doubt related to the heterogeneity of IBS. One strength of our study is that our patients with IBS were limited to 1 subtype and had to meet strict criteria for entry into our clinical trial. Our main positive finding was increased TLR4 expression in IBS, which was associated with an increase in urgency and also linked to expression of other receptors on sensory pathways.

TLRs are recognized as an important pathway whereby both microbiota and food antigens ${ }^{43}$ can activate the innate immune system. TLR4 in mice is expressed in epithelial cells and monocytes in the lamina propria, most markedly in the distal colon. ${ }^{44}$ Increased mucosal expression of TLR4 (4-5 fold), both mRNA and protein, localized to inflamed, ulcerated mucosa has been reported in children with IBD ${ }^{45}$ Lesser increases of both TLR4 and TLR5 mRNA and protein were reported in 26 patients with IBS (6 having IBS-D $)^{46}$ and of TLR2 and TLR4 $\mathrm{mRNA}$ and protein in 48 patients with IBS. ${ }^{47}$ The larger Belmont study ${ }^{47}$ reported significant differences by subtype with a 2 -fold increase in IBS-M, greater than in IBS-D, while Shukla et $\mathrm{al}^{34}$ found the greatest increase in IBS-D. We only studied IBS-D but it would be of interest to repeat these studies in a broader group of IBS including all subtypes to clarify this.

The increased expression of TLRs may underlie the excessive reaction to bacterial LPS of peripheral blood monocytes with increased secretion of key cytokines like TNF- $\alpha$, IL- 6 , and IL$1 \beta$, seen in unselected IBS, ${ }^{48}$ in patients studied 6 months after Campylobacter jejuni infection and in patients with IBS. ${ }^{49}$ Recently it has been reported that IBS-D has enhanced TLR2 and TLR4 expression which correlates with reduction of Bacteroidetes and Clostridium suggesting that increased expression may be a response to the disturbed microbiota. ${ }^{50}$

Interest in TLR activation has further been stimulated by the demonstration, using confocal laser endomicroscopy, that there are antigens in wheat, milk, and soy which can activate intra-epithelial lymphocytes and eosinophils in food intolerant patients with IBS, leading to increased small bowel permeability. ${ }^{43}$ The commonest food antigens implicated are wheat amylase/trypsin inhibitors, ${ }^{51}$ which can elicit a cytokine cascade by activating TLR4 in dendritic cells and macrophages. Our findings suggest that patients with IBS may be primed to overreact to molecules in the gut which signal via the TLR system. How this increase is driven is unclear, but in Sprague-Dawley rats subject to maternal deprivation, a stress which is known to induce anxiety and IBS-like features, increased expression of TLR3, 4, 5, and 8 has been demonstrated. ${ }^{52}$ Whether or not the associated increased TLR4 in humans with IBS is due to such a reaction is worthy of further exploration. We found increased TLR4 expression was associated with increased days with urgency, 
supporting others' findings that TLR4 expression correlated with stool frequency. ${ }^{34}$ Furthermore, in a rat model mimicking IBS, with impaired gut barrier function and increased sensitivity to colorectal distension induced by a high fermentable oligosaccharides, disaccharides, monosaccharides, and polyols (FODMAP) diet, fecal supernatants induced visceral hypersensitivity. This was blocked by small interfering RNA inhibition of TLR4 $\mathrm{mRNA}$, suggesting that TLR4 activation by fecal LPS could mediate visceral hypersensitivity. ${ }^{53}$ Animal studies using the dextran sulfate sodium colitis model $^{54}$ do show that mesalazine reduces TLR4 expression associated with its anti-inflammatory effect. However, the lack of a significant difference from placebo in our patients suggests that mesalazine only reduces TLR4 expression in the presence of active inflammation and that this reduction is likely due to the broad antiinflammatory properties of mesalazine.

In this study, we observed highly significant positive correlations between TLR4 and three other cell surface receptors; CMKLR1, TRPA1, and BDKRB2 as well as the adaptor protein MyD88. TLR4 activation has been shown to regulate gastrointestinal motility in response to gut microbial products, ${ }^{55}$ while in IBD, TLR4 signaling is thought to influence the intestinal microbiota by altering gastrointestinal motility, which drives clearance of pathogens and maintenance of commensal populations. ${ }^{56}$ It also mediates the production of defensins in response to LPS. ${ }^{57}$ Activation of TRPA1 also increases gastrointestinal motility, ${ }^{58}$ potentially as part of an important defence strategy against infection, leading to pathogen expulsion from the gut. Bradykinin is an important allogenic peptide that induces mechanical hyperalgesia via its receptor $\mathrm{BDKRB} 2{ }^{59}$ which has been shown to contribute to the sensitivity of visceral sensory neurons to noxious chemical and mechanical stimuli in both mouse and human gut tissues. ${ }^{60,61}$ Thus its upregulation may well contribute to the hypersensitivity to distension which is so characteristic of IBS. CMKLR1, the receptor for chemerin, is expressed on epithelial cells and chemerin/CMKLR1 signaling influences metabolic processes through effects on the gut microbiome. ${ }^{62}$ Its expression is also known to be important in controlling the maturation of macrophages and the balance between pro- and anti-inflammatory mucosal responses, but predicting how increased expression may alter the mucosal properties needs further study. FABP2 has been used as a marker of mucosal damage while F2RL2 and F2RL3 are protease activated receptors. Whether their upregulation would increase sensitivity to fecal proteases is unknown. Others have shown fecal proteases are increased in IBS- $\mathrm{D}^{35}$ and we have shown them to correlate with fecal urgency ${ }^{36}$ so this might be important. TRPA1 agonists have also been shown to in- crease pain in experimental models of colitis. ${ }^{63,64}$ Taken together, it is possible that increased levels of these receptors in patients with IBSD may lead to increased sensitivity to external stimuli leading to symptoms even in the absence of any overt inflammation. Although MyD88 was correlated with TLR4, it was not elevated in IBS suggesting that other factors are important in controlling its expression. One unexpected finding was the rise in TJP1. Whether this reflects a compensatory response to other causes of impaired barrier function that others have reported in IBS- $\mathrm{D}^{8-10}$ is unclear but one earlier small study suggested that this may be the case. ${ }^{65}$

While studies of microbiota in IBS are conflicting owing to lack of control of important factors like diet and antibiotic use, a metaanalysis suggests that increased numbers of Proteobacteria, including Enterobacteriaceae, and Bacteroidetes, such as Bacteroides, are a feature. Patients with IBS of either mixed or diarrhea subtype will have episodic rapid transit, which is likely to disturb the anaerobic colonic environment, disadvantaging the normally dominant anaerobes thus allowing overgrowth of the facultative, non-fastidious bacteria like Enterobacteriaceae. We showed that, as suggested by association studies of drugs and microbiota, ${ }^{1920}$ mesalazine has only modest effects on the microbial composition in patients with IBS whose mucosa shows little evidence of inflammation. The bacterial taxa reduced by the treatment, Flavonifractor and Sutterella, were not typical potential pathogens and the changes observed could be due secondary to acceleration of gut transit, since loose stools are a recognized side effect of 5 -aminosalicylic acid preparations. ${ }^{66}$

Flavonifractor has been previously linked to IBS, being increased in children with IBS in whom the amount of Flavonifractor was negatively associated with abdominal pain. ${ }^{67}$ Another study reported this taxon was decreased in patients with IBS after increased rye fiber consumption, ${ }^{68}$ but similar to the current study, there was no association to pain. Interestingly elevated levels of Flavonifractor were also found in the rectal mucosa biopsies of autistic children with gastrointestinal disorders and pain. ${ }^{69}$ Flavonifractor can degrade flavonoids and polyphenolic compounds thereby increasing their bioavailability and beneficial effect on carbohydrate metabo$\operatorname{lism}^{70}$ but how this impacts on IBS symptoms remains obscure.

Olaisen and colleagues ${ }^{13}$ have also reported a reduction in faecal Sutterella due to mesalazine treatment. Sutterella is known to inhabit the small intestine, more proximally than distal, apparently acting as a harmless commensal. ${ }^{71}$ Its abundance in feces is increased by psyllium treatment which increases colonic water. ${ }^{72} \mathrm{We}$ observed a positive correlation between Betaproteobacteria, the phylum to which Sutterella belongs, and TLR4 expression, though it is unclear if this reflects cause or effect. However, in a mouse model 
overexpression of TLR4 resulted in increased levels of mucosal Proteobacteria, including Betaproteobacteria ${ }^{73}$ suggesting TLR4 expression in mice does influence the microbiota directly.

Previous observational studies showed that high mesalazine concentrations in the mucosa were associated with reduced inflammation in ulcerative colitis ${ }^{74,75}$ and correlated with increased diversity of the mucosa associated microbiota. ${ }^{13}$ However, there was no such relationship between mucosal mesalazine concentration and fecal microbiota, which is more relevant to our study. This suggests that the widespread changes in mucosal microbiota previously reported with mesalazine treatment are mostly secondary to suppression of inflammation but that the effect on some, such as Sutterella, could be a direct effect of mesalazine.

Our study shows clearly that, in well-defined patients with IBS-D who lack histological or gene expression evidence of mucosal inflammation, the anti-inflammatory agent mesalazine neither improves symptoms nor dramatically alters inflammatory gene expression nor fecal microbiota. We have in contrast shown that many genes controlling receptors influencing responsiveness to external stimuli are upregulated and these may underlie the visceral hypersensitivity so typical of patients with IBS.

\section{Supplementary Materials}

Note: To access the supplementary Tables, Figures, and Method mentioned in this article, visit the online version of Journal of Neurogastroenterology and Motility at http://www.jnmjournal.org/, and at https://doi.org/10.5056/jnm20205.

Acknowledgements: : We would like to thank the staff of the Nottingham Biomedical Research Unit for supporting this study and Dr Abed Zaitoun and the staff of the Histopathology Department, Nottingham University Hospitals NHS Trust who performed the immunohistochemistry. This is a summary of independent research funded by the National Institute for Health Research Biomedical Research Unit. The views expressed are those of the author(s) and not necessarily those of the NHS, the NIHR, or the Department of Health.

Financial support: The clinical trial was funded by the Efficacy and Evaluation Mechanism (EME) programme (Grant No. 0920-16). Ferring Pharmaceuticals Limited sponsored the trial drug and placebo. Jonna Jalanka is funded by the Academy of Finland (316338) and Andrew Bennett by FRAME.

Conflicts of interest: Jonna Jalanka has received research grants from Sanfo-Aventis and Zespri International and speaker fees from Alfawasserman. The other authors have nothing to declare.

Author contributions: Guarantor of article: Jonna Jalanka; Ching Lam performed the trial; Anna Hartikainen, Fiona Crispie, Laura A Finnegan, Paul D Cotter, and Ching Lam performed laboratory analysis; Jonna Jalanka, Robin Spiller, Andrew Bennett, and Ching Lam analyzed the data; Robin Spiller, Ching Lam, Jonna Jalanka, and Andrew Bennett designed the study; Jonna Jalanka, Robin Spiller, Ching Lam, and Andrew Bennett wrote the paper; and all authors approved the final version of the article.

\section{References}

1. Kane JS, Irvine AJ, Derwa Y, Rotimi O, Ford AC. High prevalence of irritable bowel syndrome-type symptoms in microscopic colitis: implications for treatment. Therap Adv Gastroenterol 2018;11:1756284818783600.

2. Aziz I, Kurien M, Sanders DS, Ford AC. Screening for bile acid diarrhoea in suspected irritable bowel syndrome. Gut 2015;64:851.

3. Dunlop SP, Jenkins D, Neal KR, Spiller RC. Relative importance of enterochromaffin cell hyperplasia, anxiety, and depression in postinfectious IBS. Gastroenterology 2003;125:1651-1659.

4. Spiller R, Garsed K. Postinfectious irritable bowel syndrome. Gastroenterology 2009;136:1979-1988.

5. Jalanka J, Salonen A, Fuentes S, de Vos WM. Microbial signatures in post-infectious irritable bowel syndrome--toward patient stratification for improved diagnostics and treatment. Gut Microbes 2015;6:364-369.

6. Casén C, Vebø HC, Sekelja M, et al. Deviations in human gut microbiota: a novel diagnostic test for determining dysbiosis in patients with IBS or IBD. Aliment Pharmacol Ther 2015;42:71-83.

7. Ghoshal UC, Rahman MM. Post-infection irritable bowel syndrome in the tropical and subtropical regions: Vibrio cholerae is a new cause of this well-known condition. Indian J Gastroenterol 2019;38:87-94.

8. Dunlop SP, Hebden J, Campbell E, et al. Abnormal intestinal permeability in subgroups of diarrhea-predominant irritable bowel syndromes. Am J Gastroenterol 2006;101:1288-1294.

9. Martínez C, Lobo B, Pigrau M, et al. Diarrhoea-predominant irritable bowel syndrome: an organic disorder with structural abnormalities in the jejunal epithelial barrier. Gut 2013;62:1160-1168.

10. Vicario M, González-Castro AM, Martínez C, et al. Increased humoral immunity in the jejunum of diarrhoea-predominant irritable bowel syndrome associated with clinical manifestations. Gut 2015;64:1379-1388.

11. Martínez C, Rodiño-Janeiro BK, Lobo B, et al. miR-16 and miR-125b are involved in barrier function dysregulation through the modulation of claudin-2 and cingulin expression in the jejunum in IBS with diarrhoea. Gut 2017;66:1537-1538.

12. Xu J, Chen N, Wu Z, et al. 5-aminosalicylic acid alters the gut bacterial microbiota in patients with ulcerative colitis. Front Microbiol 2018;9:1274.

13. Olaisen M, Spigset O, Flatberg A, et al. Mucosal 5-aminosalicylic acid 
concentration, drug formulation and mucosal microbiome in patients with quiescent ulcerative colitis. Aliment Pharmacol Ther 2019;49:13011313.

14. Dahl JU, Gray MJ, Bazopoulou D, et al. The anti-inflammatory drug mesalamine targets bacterial polyphosphate accumulation. Nat Microbiol 2017;2:16267.

15. Wright EK, Kamm MA, Teo SM, Inouye M, Wagner J, Kirkwood $\mathrm{CD}$. Recent advances in characterizing the gastrointestinal microbiome in Crohn's disease: a systematic review. Inflamm Bowel Dis 2015;21:12191228.

16. Litvak Y, Byndloss MX, Bäumler AJ. Colonocyte metabolism shapes the gut microbiota. Science 2018;362:eaat9076.

17. Lloyd-Price J, Arze C, Ananthakrishnan AN, et al. Multi-omics of the gut microbial ecosystem in inflammatory bowel diseases. Nature 2019;569:655-662.

18. Andrews CN, Griffiths TA, Kaufman J, Vergnolle N, Surette MG, Rioux KP. Mesalazine (5-aminosalicylic acid) alters faecal bacterial profiles, but not mucosal proteolytic activity in diarrhoea-predominant irritable bowel syndrome. Aliment Pharmacol Ther 2011;34:374:383.

19. Maier L, Pruteanu M, Kuhn M, et al. Extensive impact of non-antibiotic drugs on human gut bacteria. Nature 2018;555:623-628.

20. Falony G, Joossens M, Vieira-Silva S, et al. Population-level analysis of gut microbiome variation. Science 2016;352:560-564.

21. Lam C, Tan W, Leighton M, et al. A mechanistic multicentre, parallel group, randomised placebo-controlled trial of mesalazine for the treatment of IBS with diarrhoea (IBS-D). Gut 2016;65:91-99.

22. Garsed K, Chernova J, Hastings M, et al. A randomised trial of ondansetron for the treatment of irritable bowel syndrome with diarrhoea. Gut 2014;63:1617-1625.

23. Schmittgen TD, Livak KJ. Analyzing real-time PCR data by the comparative C(T) method. Nat Protoc 2008;3:1101-1108.

24. Salonen A, Nikkilä J, Jalanka-Tuovinen J, et al. Comparative analysis of fecal DNA extraction methods with phylogenetic microarray: effective recovery of bacterial and archaeal DNA using mechanical cell lysis. J Microbiol Methods 2010;81:127-134.

25. Santiago A, Panda S, Mengels G, et al. Processing faecal samples: a step forward for standards in microbial community analysis. BMC Microbiol 2014;14:112.

26. Klindworth A, Pruesse E, Schweer T, et al. Evaluation of general 16S ribosomal RNA gene PCR primers for classical and next-generation sequencing-based diversity studies. Nucleic Acids Res 2013;41:e1.

27. Korpela K. Microbiota analysis in R easily. R package version 1.0. Available from URL: https://github.com/katrikorpela/mare (assessed 7 march, 2021).

28. Pinheiro JB. nlme: linear and nonlinear mixed effects models. R package version 3.1-141. Available from URL: https://CRAN.R-project.org/ package $=$ nlme (assessed 7 march, 2021).

29. Venables WR. BD. Modern applied statistics with S. New York: Springer Science \& Business Media. 2002;200:183-206.

30. Spiller RC, Humes DJ, Campbell E, et al. The patient fealth questionnaire 12 somatic symptom scale as a predictor of symptom severity and consulting behaviour in patients with irritable bowel syndrome and symp- tomatic diverticular disease. Aliment Pharmacol Ther 2010;32:811-820.

31. Foley S, Garsed K, Singh G, et al. Impaired uptake of serotonin by platelets from patients with irritable bowel syndrome correlates with duodenal immune activation. Gastroenterology 2011;140:1434-1443, e1.

32. Bashashati M, Moossavi S, Cremon C, et al. Colonic immune cells in irritable bowel syndrome: a systematic review and meta-analysis. Neurogastroenterol Motil 2018;30.

33. Vivinus-Nébot M, Frin-Mathy G, Bzioueche H, et al. Functional bowel symptoms in quiescent inflammatory bowel diseases: role of epithelial barrier disruption and low-grade inflammation. Gut 2014;63:744-752.

34. Shukla R, Ghoshal U, Ranjan P, Ghoshal UC. Expression of toll-like receptors, pro-, and anti-inflammatory cytokines in relation to gut microbiota in irritable bowel syndrome: the evidence for its micro-organic basis. J Neurogastroenterol Motil 2018;24:628-642.

35. Gecse K, Róka R, Ferrier L, et al. Increased faecal serine protease activity in diarrhoeic IBS patients: a colonic lumenal factor impairing colonic permeability and sensitivity. Gut 2008;57:591-599.

36. Tooth D, Garsed K, Singh G, et al. Characterisation of faecal protease activity in irritable bowel syndrome with diarrhoea: origin and effect of gut transit. Gut 2014;63:753-760.

37. Buhner S, Hahne H, Hartwig K, et al. Protease signaling through protease activated receptor 1 mediate nerve activation by mucosal supernatants from irritable bowel syndrome but not from ulcerative colitis patients. PLoS One 2018;13:e0193943.

38. Barbara G, Cremon C, Annese V, et al. Randomised controlled trial of mesalazine in IBS. Gut 2016;65:82-90.

39. Camilleri M, Carlson P, Acosta A, et al. RNA sequencing shows transcriptomic changes in rectosigmoid mucosa in patients with irritable bowel syndrome-diarrhea: a pilot case-control study. Am J Physiol Gastrointest Liver Physiol 2014;306:G1089-G1098.

40. Videlock EJ, Mahurkar-Joshi S, Hoffman JM, et al. Sigmoid colon mucosal gene expression supports alterations of neuronal signaling in irritable bowel syndrome with constipation. Am J Physiol Gastrointest Liver Physiol 2018;315:G140-G157.

41. Camilleri M, Carlson P, Acosta A, Busciglio I. Colonic mucosal gene expression and genotype in irritable bowel syndrome patients with normal or elevated fecal bile acid excretion. Am J Physiol Gastrointest Liver Physiol 2015;309:G10-G20.

42. Zhao JH, Dong L, Shi HT, Wang ZY, Shi HY, Ding H. The expression of protease-activated receptor 2 and 4 in the colon of irritable bowel syndrome patients. Dig Dis Sci 2012;57:58-64.

43. Fritscher-Ravens A, Pflaum T, Mösinger M, et al. Many patients with irritable bowel syndrome have atypical food allergies not associated with immunoglobulin E. Gastroenterology 2019;157:109-118, e5.

44. Ortega-Cava CF, Ishihara S, Rumi MA, et al. Strategic compartmentalization of toll-like receptor 4 in the mouse gut. J Immunol 2003;170:3977-3985.

45. Szebeni B, Veres G, Dezsõfi A, et al. Increased expression of toll-like receptor (TLR) 2 and TLR4 in the colonic mucosa of children with inflammatory bowel disease. Clin Exp Immunol 2008;151:34-41.

46. Brint EK, Macsharry J, Fanning A, Shanahan F, Quigley EM. Differential expression of toll-like receptors in patients with irritable bowel 
syndrome. Am J Gastroenterol 2011;106:329-336.

47. Belmonte L, Beutheu Youmba S, Bertiaux-Vandaële N, et al. Role of toll like receptors in irritable bowel syndrome: differential mucosal immune activation according to the disease subtype. PLoS One 2012;7:e42777.

48. Liebregts T, Adam B, Bredack C, et al. Immune activation in patients with irritable bowel syndrome. Gastroenterology 2007;132:913-920.

49. Swan C, Duroudier NP, Campbell E, et al. Identifying and testing candidate genetic polymorphisms in the irritable bowel syndrome (IBS): association with TNFSF15 and TNFalpha. Gut 2013;62:985-994.

50. Guo WT, Liu P, Dong LN, Wang JP. [The correlation study between the changes of intestinal mucosa predominant bacteria and toll-like receptor 2, toll-like receptor 4 gene expressions in diarrhea-predominant irritable bowel syndrome patients.] Zhonghua Nei Ke Za Zhi 2016;55:541543. [Chinese]

51. Pickert G, Wirtz S, Matzner J, et al. Wheat consumption aggravates colitis in mice via amylase trypsin inhibitor-mediated dysbiosis. Gastroenterology 2020;159:257-272, e17.

52. McKernan DP, Nolan A, Brint EK, et al. Toll-like receptor mRNA expression is selectively increased in the colonic mucosa of two animal models relevant to irritable bowel syndrome. PLoS One 2009;4:e8226.

53. Zhou SY, Gillilland M 3rd, Wu X, et al. FODMAP diet modulates visceral nociception by lipopolysaccharide-mediated intestinal inflammation and barrier dysfunction. J Clin Invest 2018;128:267-280.

54. Feng J, Guo C, Zhu Y, et al. Baicalin down regulates the expression of TLR4 and NFkB-p65 in colon tissue in mice with colitis induced by dextran sulfate sodium. Int J Clin Exp Med 2014;7:4063-4072.

55. Anitha M, Vijay-Kumar M, Sitaraman SV, Gewirtz AT, Srinivasan S. Gut microbial products regulate murine gastrointestinal motility via tolllike receptor 4 signaling. Gastroenterology 2012;143:1006-1016, e4.

56. Frosali S, Pagliari D, Gambassi G, Landolfi R, Pandolfi F, Cianci R. How the intricate interaction among toll-like receptors, microbiota, and intestinal immunity can influence gastrointestinal pathology. J Immunol Res 2015;2015:489821.

57. Vora P, Youdim A, Thomas LS, et al. Beta-defensin-2 expression is regulated by TLR signaling in intestinal epithelial cells. J Immunol 2004;173:5398-5405.

58. Nozawa K, Kawabata-Shoda E, Doihara H, et al. TRPA1 regulates gastrointestinal motility through serotonin release from enterochromaffin cells. Proc Natl Acad Sci USA 2009;106:3408-3413.

59. Mizumura K, Sugiura T, Katanosaka K, Banik RK, Kozaki Y. Excitation and sensitization of nociceptors by bradykinin: what do we know? Exp Brain Res 2009;196:53-65.

60. Brierley SM, Jones RC 3rd, Xu L, Gebhart GF, Blackshaw LA. Activation of splanchnic and pelvic colonic afferents by bradykinin in mice. Neurogastroenterol Motil 2005;17:854-862.

61. Wang S, Dai Y, Fukuoka T, et al. Phospholipase C and protein kinase A mediate bradykinin sensitization of TRPA1: a molecular mechanism of inflammatory pain. Brain 2008;131(Pt 5):1241-1251.

62. Dranse HJ, Zheng A, Comeau AM, Langille MGI, Zabel BA, Sinal CJ. The impact of chemerin or chemokine-like receptor 1 loss on the mouse gut microbiome. PeerJ 2018;6:e5494.

63. Brierley SM, Hughes PA, Page AJ, et al. The ion channel TRPA1 is required for normal mechanosensation and is modulated by algesic stimuli. Gastroenterology 2009;137:2084-2095, e3.

64. Peiris M, Hockley JR, Reed DE, Smith ESJ, Bulmer DC, Blackshaw LA. Peripheral KV7 channels regulate visceral sensory function in mouse and human colon. Mol Pain 2017;13:1744806917709371.

65. Camilleri M, Carlson P, Valentin N, et al. Pilot study of small bowel mucosal gene expression in patients with irritable bowel syndrome with diarrhea. Am J Physiol Gastrointest Liver Physiol 2016;311:G365-G376.

66. Wang Y, Parker CE, Bhanji T, Feagan BG, MacDonald JK. Oral 5-aminosalicylic acid for induction of remission in ulcerative colitis. Cochrane Database Syst Rev 2016;4:CD000543.

67. Hollister EB, Oezguen N, Chumpitazi BP, et al. Leveraging human microbiome features to diagnose and stratify children with irritable bowel syndrome. J Mol Diagn 2019;21:449-461.

68. Laatikainen R, Jalanka J, Loponen J, et al. Randomised clinical trial: effect of low-FODMAP rye bread versus regular rye bread on the intestinal microbiota of irritable bowel syndrome patients: association with individual symptom variation. BMC Nutr 2019;5:12.

69. Ribas Y, Aguilar F, Jovell-Fernández E, Cayetano L, Navarro-Luna A, Muñoz-Duyos A. Clinical application of the LARS score: results from a pilot study. Int J Colorectal Dis 2017;32:409-418.

70. Moco S, Martin FP, Rezzi S. Metabolomics view on gut microbiome modulation by polyphenol-rich foods. J Proteome Res 2012;11:47814790.

71. Hiippala K, Kainulainen V, Kalliomäki M, Arkkila P, Satokari R. Mucosal prevalence and interactions with the epithelium indicate commensalism of Sutterella spp. Front Microbiol 2016;7:1706.

72. Jalanka J, Major G, Murray K, et al. The effect of psyllium husk on intestinal microbiota in constipated patients and healthy controls. Int $\mathrm{J}$ Mol Sci 2019;20:433.

73. Dheer R, Santaolalla R, Davies JM, et al. Intestinal epithelial toll-like receptor 4 signaling affects epithelial function and colonic microbiota and promotes a risk for transmissible colitis. Infect Immun 2016;84:798-810.

74. Frieri G, Giacomelli R, Pimpo M, et al. Mucosal 5-aminosalicylic acid concentration inversely correlates with severity of colonic inflammation in patients with ulcerative colitis. Gut 2000;47:410-414.

75. Naganuma M, Iwao Y, Ogata H, et al. Measurement of colonic mucosal concentrations of 5-aminosalicylic acid is useful for estimating its therapeutic efficacy in distal ulcerative colitis: comparison of orally administered mesalamine and sulfasalazine. Inflamm Bowel Dis 2001;7:221-225. 